Article

\title{
Investigating the Effect of Individual Rotations on the Physical and Physiological Performance in Elite Female Field Hockey Players
}

\author{
Aideen McGuinness ${ }^{1, *}$, Darren Kenna ${ }^{2}$, Adam Grainger ${ }^{3}\left[\right.$ and Kieran Collins ${ }^{1}$ \\ 1 Tallaght Campus, Technological University of Dublin, Tallaght, D24 FKT9 Dublin, Ireland; \\ Kieran.Collins@tudublin.ie \\ 2 Irish Strength Institute, Malahide Rd, Artane, D05 W6P2 Dublin, Ireland; darren@ueperformancetherapy.com \\ 3 Hockey Ireland, Richview Newstead Block C, Stillorgan Rd, Belfield, D04 V1W8 Dublin, Ireland; \\ Adam.Grainger@hockey.ie \\ * Correspondence: mcguinnessaideen@gmail.com; Tel.: +353-83-181-9630
}

Citation: McGuinness, A.; Kenna, D. Grainger, A.; Collins, K. Investigating the Effect of Individual Rotations on the Physical and Physiological Performance in Elite Female Field Hockey Players. Appl. Sci. 2021, 11, 1022. https://doi.org/10.3390/ app11031022

Received: 21 December 2020

Accepted: 17 January 2021

Published: 23 January 2021

Publisher's Note: MDPI stays neutral with regard to jurisdictional claims in published maps and institutional affiliations.

Copyright: (c) 2021 by the authors. Licensee MDPI, Basel, Switzerland. This article is an open access article distributed under the terms and conditions of the Creative Commons Attribution (CC BY) license (https:// creativecommons.org/licenses/by/ $4.0 /)$.

\begin{abstract}
To quantify the rotational demands of elite female field hockey with respect to position. Twenty-eight $(n=28)$ elite international field hockey players were recruited during the 2018-2020 seasons. Players were monitored with GPS technology and heart-rate monitors. Methods: Activity was categorised into total distance $(\mathrm{m})$, relative total distance $\left(\mathrm{m} \cdot \mathrm{min}^{-1}\right)$, high-speed distance $(\mathrm{m}$; $\left.16 \mathrm{~km} \cdot \mathrm{h}^{-1}\right)$, relative high-speed distance $\left(\mathrm{m} \cdot \mathrm{min}^{-1}\right)$, max velocity $\left(\mathrm{km} \cdot \mathrm{h}^{-1}\right)$, and percentage maximal velocity (\%Vmax). Physiological demands were assessed via heart rate measures (bpm and \% $\mathrm{HR}_{\max }$ ) and time $>80 \%$ heart rate maximum. Results: A single rotation equated to $7 \pm 0.8$ min. Players covered a mean total distance of was $868 \pm 132 \mathrm{~m}\left(125.7 \pm 5.9 \mathrm{~m} \cdot \mathrm{min}^{-1}\right)$ with $140 \pm 39 \mathrm{~m}$ at highspeed $\left(21.7 \pm 3.6 \mathrm{~m} \cdot \mathrm{min}^{-1}\right)$. A significant difference was reported for relative total $(p \leq 0.001)$, and high-speed ( $p \leq 0.001)$, distance across positional. Forwards were reported to cover the most relative total and high-speed distance $(d=1.0)$ when compared to defenders and midfielders. Conclusion: The study provides normative data for rotational demands of elite female field hockey. Coaches should consider these demands when developing training drills to better optimise the positional physical and physiological demands of competitive match-play.
\end{abstract}

Keywords: team sport; GPS; intermittent exercise; running performance; high intensity work-rate

\section{Introduction}

Field hockey can be best described as a stick and ball field-based sport comprised of both technical and tactical components that are completed at moderate to high speeds [1-3]. In recent years, field hockey has undergone vast technical and structural changes. One of these significant changes occurred in 2016 when the structure of the game went from two halves to four quarters. Match-play now consists of four 15 min quarters (Q's) with a 2 min break separating quarters 1 and 2 and quarters 3 and 4 with half-time consisting of a 10 min break separating quarters 2 and 3. However, in 1992, the International hockey federation $(\mathrm{FIH})$ introduced the "rolling substitutions" rule whereby substitutions could take place at any time during the game. These are known as "rotations" by coaching and performance staff. A total of eighteen players are eligible to play during match-play: two keepers and 16 outfield players. During match-play, 11 players may be on the pitch at one time with a total of five additional players available to roll-on and roll-off at any time. Anecdotally, players will perform 6-8 rotations during a game with the timing of these rotations pre-determined based on the tactical decisions of the Head Coach.

Research focused on elite female hockey match-play has increased substantially, with these studies reporting that typically players cover on average $4847 \pm 58-\mathrm{m}(127.6 \pm$ $\left.15.3 \mathrm{~m} \cdot \mathrm{min}^{-1}\right)$ with $580 \pm 147-\mathrm{m}\left(15.3 \pm 3.9 \mathrm{~m} \cdot \mathrm{min}^{-1}\right)$ performed above $16 \mathrm{~km} \cdot \mathrm{h}^{-1}$ [3-8]. 
McMahon and Kennedy [7] were the first to report the changes in player's activity profiles following structural changes to competitive match-play-a significant increase in relative total $\left(7.5 \mathrm{~m} \cdot \mathrm{min}^{-1}, d=0.6\right)$ and high-speed distance $\left(0.6 \mathrm{~m} \cdot \mathrm{min}^{-1}, d=0.3\right)$. Furthermore, a substantial decrease in low-speed relative distance $\left(-2.2 \mathrm{~m} \cdot \mathrm{min}^{-1}, d=0.4\right)$ during matchplay was noted in conjunction with these rule changes. In recent years, McGuinness et al. (2018) observed changes in the running performance of elite female hockey players across the four quarters of match-play. Within this literature, defenders $(5181 \pm 607 \mathrm{~m})$ were shown to cover the most total distance, meanwhile it was reported that midfielders cover the most distance at moderate intensity $(1313 \pm 173 \mathrm{~m})$. However, no differences were reported for relative distance across quarters of play. Significant differences were observed for relative high-speed distance across quarters with a significant drop from quarter 1 to quarter 2 and further reductions between quarter 2 and quarter 4 . When positions were considered, forwards were observed to cover the most relative distance at high-speed $\left(18.2 \pm 7.9 \mathrm{~m} \cdot \mathrm{min}^{-1}\right)$ when compared to the defenders $\left(13.8 \pm 4.8 \mathrm{~m} \cdot \mathrm{min}^{-1}\right)$ and midfielders $\left(14.8 \pm 6.2 \mathrm{~m} \cdot \mathrm{min}^{-1}\right)$.

Due to greater interest in match demands and their influence upon match outcome, practitioners have begun to monitor duration specific moments within competitive matchplay, such as rolling average and rotational running performance demands [5,6,9-12]. Previously, McGuinness et al. [6] quantified the peak running intensity of elite female field hockey players during match-play. Irrespective of position, players were reported to cover an average peak relative distance of $159-201 \mathrm{~m} \cdot \mathrm{min}^{-1}$. Forwards were observed to have the highest peak $1 \mathrm{~min}$ rolling average $\left(200 \pm 11 \mathrm{~m} \cdot \mathrm{min}^{-1}\right)$ when compared to the defenders $\left(188 \pm 16 \mathrm{~m} \cdot \mathrm{min}^{-1}\right)$ and midfielders $\left(195 \pm 15 \mathrm{~m} \cdot \mathrm{min}^{-1}\right)$. Following on from these findings, Linke and Lames [11] quantified the running performance of substitutes within elite male field hockey. During each rotation, players would cover an average relative distance of $139 \mathrm{~m} \cdot \mathrm{min}^{-1}$, with the mean distance covered during rotation $1\left(157 \mathrm{~m} \cdot \mathrm{min}^{-1}\right)$ and rotation $2\left(143 \mathrm{~m} \cdot \mathrm{min}^{-1}\right)$ decreasing significantly $(p \leq 0.05)$. As per current international rules, five players are available to be substituted during match-play, with coaches using these roll on and roll off interchanges as a method to maintain higher work-rates across all periods, understanding the demands of these interchanges would appear important from a performance and training replication perspective.

Due to the roll-on roll-off nature of these interchanges, coaching staff have begun to focus on the performance requirements of these rotations to best replicate and exceed these demands within training situations when appropriate. However, currently, there is a limited number of research studies that have focused on the rotational demands of elite female field hockey $[5,11]$. Therefore, an examination of the physical and physiological demands during each player rotation in elite female field hockey is warranted to aid coaching practice with respect to training drill creation and construction. By understanding these physical and physiological demands it will enable practitioners to create training segments that better replicate the position-specific demands of competitive match-play, allowing the coaching staff to construct duration specific small-sided games that in turn replicate the rotational demands of match-play. Given the above, the aim of the current study was to quantify the physical and physiological performance demands of elite female field hockey players with respect to rotations. Secondly, we aimed to understand these demands across the positional lines of play. It was hypothesised that forwards would have a higher relative total distance; midfielders would cover the greatest distance above $16 \mathrm{~km} \cdot \mathrm{h}^{-1}$ while the defenders would spend the most amount of time $>80 \%$ heart rare $\operatorname{maxxx}\left(\mathrm{HR}_{\max }\right)$.

\section{Materials and Methods}

\subsection{Experimental Approach to the Problem}

The current study was observational in nature and designed to quantify the physical and physiological rotational demands of elite female hockey players using GPS technology (10-Hz, STATSports Apex Unit, STATSports Group Limited, Sonra 2.1.15, Newry, Northern 
Ireland, UK) and heart rate monitors (Polar T-31, Polar Electro Oy, Kempele, Finland). Twenty-eight $(n=28)$ elite international female hockey players were observed during twenty-seven competitive matches $(n=27)$. All competitive matches that occurred during the 2018-2020 seasons were included in the data set. Overall, there were three hundred and nighty five $(n=395)$ observations included in the data set. On average, players appeared in thirteen $(13 \pm 8)$ of the twenty-seven games. Of the 27 games, 12 games were official FIH tournaments including the FIH Series Finals (5), Europeans (5) and the Olympic qualifiers (2). The rest of the games that were included in the data set were friendlies against other senior national teams that were ranked within the top 16 . In order for a friendly game to be included in the data set the following criteria must be met: (1) The game must follow the official FIH format of 4 Qs and breaks in play (2); The teams may only have 18 players on the bench -16 outfield and 2 goalkeepers; (3) The umpires must be either internationally or national recognised. All participants were categorised by the head coach into three positional lines of play: defenders $(n=8)$, midfielders $(n=9)$, and forwards $(n=11)$, respectively. If a player was to play in a position that they were not previously categorised into by the head coach, this data set was removed from the cohort of data. In order to gain a better understanding of the average rotational demands, rotational data were only included in the current study if the player spent more than $90 \%$ of the team's minimum rotation duration on the field ( 3:40 min). Prior to match-day, players were advised to abstain from any strenuous activity and were advised to maintain their normal pre-match routine and diet with special emphasis being placed on the intake of fluids and carbohydrates.

\subsection{Participants}

Twenty-eight $(n=28)$ elite international field hockey outfield players ( $26 \pm 3$ years; $162.7 \pm 12 \mathrm{~cm} ; 67.3 \pm 5.4 \mathrm{~kg}$ ) were recruited to participate in the study. At the time of the current study, the team in question was ranked 8th in the FIH world rankings and players were part of the country's Tokyo 2020 Olympic training panel $(9 \pm 4$ years at International level) and were therefore deemed the best players in the country during the observational period. Once ethical approval was granted, participants were informed of the benefits and procedures of the current study. Written informed consent and medical declaration were obtained from participants in line with the procedures set by the research ethics committee of the Technological University of Dublin (REC-PGR26-201819).

\subsection{Experimental Procedures}

During all competitive match-play situations, participants wore an individual GPS unit (10-Hz, STATSports Apex Unit, STATSports Group Limited, Newry, Northern Ireland, UK) to understand participants running performance across each rotation of play. The physiological performance was assessed through each participant wearing a HR monitor during match-play (Polar T-31, Polar Electro Oy, Kempele, Finland). Fifteen minutes prior to match-play each unit was switched on to allow the unit to establish a satellite connection. Each participant wore their own individual GPS unit within the confines of a vest between the shoulder blades. Prior to match-play, players' individual rotations were pre-determined by a member of the management team. It was each individual player's responsibility to ensure they rotated with the correct players marked on the sheet at the correct time. There was also a member of staff allocated to monitor the bench during competition to make sure players stayed on top of their rotation times. Once a player had finished their rotation, they were asked to take their water bottle and walk straight to the end line and back to the bench where they were asked to sit until it was time for their next rotation. If for any reason a player was unable to rotate with another player, the sports scientist would take note of this on the rational timings sheet. Given the use of rolling substitutes, the time each participant spent in match-play was noted by the sports scientist with the use of a customised IOS app (Coaches Clock, Version 1.3.2, USA). Upon the completion of match-play, the data were downloaded using proprietary software (Apex SONRA, STATSports, Newry, Northern Ireland, UK), with all data for respective rotations 
completed analysed retrospectively and applied to each individual play. All data were than exported to a bespoke database (Microsoft Excel (V15); Microsoft, Redmond, USA). Each individual rotation was than retrospectivity cut using the pre-determined rotational timing sheet. The sports scientist was responsible to match up the rotational times with the movement graph on the proprietary software. If at any stage, the sports scientist was unable to determine where a rotation was made, they were encouraged to use the video footage from the match. All data were then exported to a bespoke database (Microsoft Excel(V15); Microsoft, Redmond, USA).

Participants' speed thresholds were classified based on previous zones used in elite female field hockey literature [13]. Variables of interest included time on feet (min), total distance $(\mathrm{m})$, relative total distance $\left(\mathrm{m} \cdot \mathrm{min}^{-1}\right)$, high-speed distance $\left(\mathrm{m} \geq 16 \mathrm{~km} \cdot \mathrm{h}^{-1}\right)$, relative high-speed distance $\left(\mathrm{m} \cdot \mathrm{min}^{-1}\right)$, sprint distance $\left(\mathrm{m} \geq 20 \mathrm{~km} \cdot \mathrm{h}^{-1}\right)$, relative sprint distance $\left(\mathrm{m} \cdot \mathrm{min}^{-1}\right)$, no. of sprints $\left(\mathrm{n} ;>20 \mathrm{~km} \cdot \mathrm{h}^{-1}\right)$, maximal speed $\left(\mathrm{km} \cdot \mathrm{h}^{-1}\right)$, percentage maximal speed (\% Vmax), accelerations ( $\mathrm{n} ; \geq 4 \mathrm{~m} \cdot \mathrm{s}^{-2}$ ) and decelerations $\left(\mathrm{n} ; \leq-4 \mathrm{~m} \cdot \mathrm{s}^{-2}\right.$ ). Physiological demands were also assessed during match-play with variables of interest including $\mathrm{HR}_{\max }(\mathrm{bpm}), \mathrm{HR}_{\max }(\%)$, time $\geq 80 \% \mathrm{HR}_{\max }$ (min: ss). Prior to the observational period, players completed the Yo-YoIR1 to determine each participant's $H_{\max }$. The highest heart rate obtained by the participant during this test was deemed to be the player $\mathrm{HR}_{\max }$. However, if a participant obtained a higher $\mathrm{HR}_{\max }$ throughout the course of testing, this figure would then be replaced and all data prior to this reading were then re-analysed $(n=2)$. If at any stage an athlete's heart rate data did not record, that specific rotation was omitted for analysis.

\subsection{Statistical Analysis}

Data are presented as a mean \pm standard deviation (SD) with $95 \%$ confidence intervals ( $95 \%$ CIs) and partial Eta-squared $\left(\eta^{2}\right)$ unless stated otherwise. Multiple two-way analyses of variance (ANOVAs) were used to determine the potential differences in locomotor activity and physiological variables across positional groups $(n=3)$ and for each rotation. Significance was set at $p \leq 0.05$. When significant main effects were observed, GamesHowell post-hoc test was applied. Partial Eta-squared standardised effect sizes (ES) were defined as $\leq 0.01$, small; $>0.01-0.06$, medium; $>0.06-0.14$, large [14]. Cohens d standardised ES were defined as $\leq 0.2$, small; $>0.2-0.5$, medium; $>0.5-0.8$, large, $\geq 0.8$, very large [15]. Where an effect size Cohen's $d$ of $>0.5$ (medium) was observed, it was flagged as a potential difference. All statistical analyses were performed using SPSS for Windows (Version 22, SPSS Inc., Chicago, IL, USA).

\section{Results}

\subsection{Physical Performance Demands across Rotations}

Selected running variables are presented in Tables 1 and 2 . When total distance was observed, no significant interaction was reported between position $(\mathrm{F}(8,1766)=0.08$; $p=0.9 ; \eta^{2}=0.001 ;$ small $)$ and rotation $\left(\mathrm{F}=(8,1766)=0.5 ; p=0.8 ; \eta^{2}=0.002 ;\right.$ small $)$. A significant interaction between position $\left(\mathrm{F}(2,541)=9.8 ; p \leq 0.001, \eta^{2}=0.05\right.$, small $)$ and high-speed distance $(\mathrm{m})$. However, when each rotation was considered, no interaction was reported $\left(\mathrm{F}(8,1766)=2 ; p=0.7 ; \eta^{2}=0.008\right.$; small). When positions were considered, a significant main effect was observed. Defenders were reported to cover significantly less high-speed distance $(108 \pm 56)$ when compared to the midfield $(149 \pm 65 ; \mathrm{d}=0.7$; large) and forwards ( $157 \pm 73 ; \mathrm{d}=0.8$; large). Forwards were also reported to cover significantly more high-speed distance then the midfield ( $149 \pm 65 ; \mathrm{d}=1.1$; large). There was significant interaction between position $\left(\mathrm{F}(2,1766)=15 ; p \leq 0.001 ; \eta^{2}=0.02\right.$; small) and sprint distance $(\mathrm{m})$; however, when each rotation was considered, no interaction was reported $\left(\mathrm{F}(8,1766)=0.9 ; p=0.5 ; \eta^{2}=0.004 ;\right.$ small $)$. When positions were considered, a significant main effect was observed. Defenders were reported to cover significantly less sprint distance $(27 \pm 8)$ when compared to the midfield $(30 \pm 10 ; \mathrm{d}=0.3$; medium) and forwards $(34 \pm 9 ; \mathrm{d}=0.8 ;$ large $)$. The was significant interaction between position $(\mathrm{F}(2,1766)=11$; 
$p \leq 0.001 ; \eta^{2}=0.01 ;$ small) and maximal speed $\left(\mathrm{km} \cdot \mathrm{h}^{-1}\right)$; however, when each rotation was considered, no interaction was reported $\left(\mathrm{F}(8,1766)=1.0 ; p=0.5 ; \eta^{2}=0.004 ;\right.$ small $)$. Defenders $(22.9 \pm 0.5)$ were likely to achieve a lower maximal speed $\left(\mathrm{km} \cdot \mathrm{h}^{-1}\right)$ when compared to the forwards $(23.2 \pm 0.7 ; \mathrm{d}=0.5 ;$ medium $)$ and midfielders $(23.0 \pm 0.8 ; \mathrm{d}=0.2$; medium). A significant interaction was observed across position $(\mathrm{F}(2,1766)=0.5 ; p=0.04$; $\eta^{2}=0.004$; small $)$ and rotation $\left(\mathrm{F}(8,1766)=0.8 ; p=0.7 ; \eta^{2}=0.007\right.$; small $)$ for maximal speed $(\%)$. Defenders $(77 \pm 2)$ were likely to achieve a lower maximal speed $(\%)$ when compared to the forwards ( $79 \pm 2 ; \mathrm{d}=1.0$; medium) and midfielders $(79 \pm 2 ; \mathrm{d}=1.0$; medium).

\subsection{Physiological Performance Demands across Rotations}

Selected physiological demands are presented in Tables 1 and 2. When $\mathrm{HR}_{\max }(\mathrm{bpm})$ was observed, a significant interaction was reported between position $(\mathrm{F}(2,1766)=31.0$, $p \leq 0.001 ; \eta^{2}=0.03$; small); however, no significant interaction was observed across rotations $\left(\mathrm{F}(8,1766)=1.8 ; p=0.07 ; \eta^{2}=0.08\right.$; small). When positions were considered, a significant main effect was observed. Defenders were reported to have a lower $\mathrm{HR}_{\max }$ (bpm) $(183 \pm 3)$ when compared to the midfield ( $188 \pm 3 ; \mathrm{d}=1.7$; very large) and forwards $(190 \pm 3 ; d=2.3$; very large). Forwards were also reported to achieve a higher heart rate when compared to midfield ( $149 \pm 65, \mathrm{~d}=0.6$; medium). When $\% \mathrm{HR}_{\max }$ was observed, a significant interaction was reported between position $\left(\mathrm{F}(2,1766)=12.8 ; p \leq 0.001 ; \eta^{2}=0.01\right.$; small). However, no significant interaction was observed across rotations $(\mathrm{F}(8,1766)=0.07$; $p=0.7 ; \eta^{2}=0.003 ;$ small). When positions were considered a significant main effect was observed. Defenders were reported to achieve a lower $\mathrm{HR}_{\max }(\%)(86 \pm 2)$ when compared to the forwards ( $89 \pm 3 ; \mathrm{d}=1.2 ;$ large) and midfield ( $89 \pm 3 ; \mathrm{d}=1.2 ;$ large). 


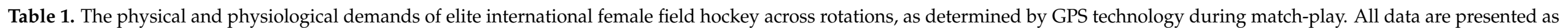
mean \pm SD.

\begin{tabular}{|c|c|c|c|c|c|c|c|c|c|c|c|c|c|c|}
\hline & & $\begin{array}{l}\text { Duration } \\
\text { (min) }\end{array}$ & $\begin{array}{c}\text { Total } \\
\text { Distance } \\
\text { (m) }\end{array}$ & $\begin{array}{c}\text { Total } \\
\text { Distance } \\
(\mathbf{m} \cdot \mathbf{m i n})\end{array}$ & $\begin{array}{c}\text { High- } \\
\text { Speed } \\
\text { Distance }\end{array}$ & $\begin{array}{c}\text { Sprint } \\
\text { Distance }\end{array}$ & $\begin{array}{l}\text { No. of } \\
\text { Accelera- } \\
\text { tions }\end{array}$ & $\begin{array}{l}\text { No. of } \\
\text { Decelera- } \\
\text { tions }\end{array}$ & $\begin{array}{c}\text { Peak } \\
\text { Speed } \\
(\mathbf{k m} \cdot \mathbf{h})\end{array}$ & $\begin{array}{l}\text { Max } \\
\text { Speed } \\
(\%)\end{array}$ & $\begin{array}{c}\text { No. } \\
\text { Sprints } \\
>20 \\
\mathrm{~km} \cdot \mathrm{h}\end{array}$ & $\begin{array}{c}\text { Peak HR } \\
\text { (bpm) }\end{array}$ & $\begin{array}{c}\mathrm{HR}_{\max } \\
(\%)\end{array}$ & $\begin{array}{c}\text { Time }> \\
80 \% \\
H^{H} R_{\max } \\
\text { (min:ss) }\end{array}$ \\
\hline \multicolumn{2}{|c|}{ Avg. Rotation } & $7: 00 \pm 0: 50$ & $868 \pm 132$ & $124.9 \pm 10.7$ & $140 \pm 39$ & $31 \pm 13$ & $5 \pm 2$ & $7 \pm 2$ & $23.0 \pm 0.9$ & $76 \pm 1$ & $2 \pm 1$ & $187 \pm 8$ & $86 \pm 4$ & $4: 20 \pm 1: 30$ \\
\hline \multicolumn{2}{|c|}{ Avg. Defender } & $7: 30 \pm 3: 15$ & $881 \pm 138$ & $119.8 \pm 11.0$ & $117 \pm 34$ & $26 \pm 11$ & $5 \pm 2$ & $7 \pm 2$ & $22.8 \pm 0.8$ & $74 \pm 3$ & $2 \pm 1$ & $189 \pm 6$ & $84 \pm 4$ & $4: 18 \pm 1: 20$ \\
\hline \multicolumn{2}{|c|}{ Avg. Midfield } & $7: 20 \pm 1: 15$ & $884 \pm 117$ & $123.6 \pm 7.6$ & $153 \pm 30$ & $33 \pm 12$ & $5 \pm 1$ & $8 \pm 2$ & $23.3 \pm 0.9$ & $78 \pm 4$ & $2 \pm 1$ & $188 \pm 6$ & $90 \pm 6$ & $4: 20 \pm 1: 30$ \\
\hline \multicolumn{2}{|c|}{ Avg. Forward } & $6: 30 \pm 2: 30$ & $836 \pm 132$ & $131.1 \pm 11$ & $160 \pm 37$ & $36 \pm 15$ & $6 \pm 2$ & $7 \pm 2$ & $23.3 \pm 0.9$ & $73 \pm 3$ & $3 \pm 1$ & $184 \pm 10$ & $86 \pm 5$ & $4: 10 \pm 2: 10$ \\
\hline $\mathrm{R} 1$ & $\begin{array}{l}\text { Team } \\
\text { Avg. }\end{array}$ & $6: 30 \pm 2: 30$ & $866 \pm 141$ & $126.7 \pm 9.5$ & $145 \pm 45$ & $31 \pm 11$ & $6 \pm 2 *$ & $8 \pm 1 *$ & $23.1 \pm 0.4$ & $78 \pm 2 *$ & $2 \pm 1$ & $188 \pm 8$ & $89 \pm 5^{*}$ & $4: 18 \pm 1: 30$ \\
\hline $\mathrm{R} 2$ & $\begin{array}{l}\text { Team } \\
\text { Avg. }\end{array}$ & $7: 00 \pm 2: 30$ & $855 \pm 99$ & $126.4 \pm 11.9$ & $136 \pm 34$ & $28 \pm 9$ & $5 \pm 2$ & $7 \pm 2$ & $23.0 \pm 0.3$ & $78 \pm 2 *$ & $2 \pm 1$ & $189 \pm 7$ & $89 \pm 5^{*}$ & $4: 34 \pm 1: 30$ \\
\hline R3 & $\begin{array}{l}\text { Team } \\
\text { Avg. }\end{array}$ & $7: 00 \pm 2: 30$ & $837 \pm 109$ & $124.2 \pm 10.7$ & $132 \pm 42^{1}$ & $28 \pm 14$ & $5 \pm 2$ & $7 \pm 2$ & $22.8 \pm 1.6$ & $78 \pm 3 *$ & $2 \pm 1$ & $189 \pm 7$ & $89 \pm 5^{*}$ & $4: 21 \pm 1: 20$ \\
\hline $\mathrm{R} 4$ & $\begin{array}{l}\text { Team } \\
\text { Avg. }\end{array}$ & $7: 30 \pm 2: 55$ & $874 \pm 106$ & $119.8 \pm 106$ & $131 \pm 31^{1}$ & $29 \pm 11$ & $5 \pm 2$ & $7 \pm 2$ & $23.0 \pm 0.4$ & $78 \pm 3 *$ & $2 \pm 1$ & $188 \pm 8$ & $89 \pm 5$ & 4: $23 \pm 2: 00$ \\
\hline R6 & $\begin{array}{l}\text { Team } \\
\text { Avg. }\end{array}$ & $8: 00 \pm 3: 00$ & $891 \pm 131$ & $118.8 \pm 8.9$ & $131 \pm 41^{1,2}$ & $31 \pm 13$ & $5 \pm 2$ & $7 \pm 2$ & $23.0 \pm 1.2$ & $78 \pm 3^{*}$ & $2 \pm 1$ & $188 \pm 8$ & $89 \pm 5$ * & $4: 39 \pm 2: 10$ \\
\hline R7 & $\begin{array}{l}\text { Team } \\
\text { Avg. }\end{array}$ & $7: 30 \pm 3: 20$ & $840 \pm 209$ & $120.7 \pm 9.4$ & $135 \pm 45^{1}$ & $32 \pm 19$ & $6 \pm 2 *$ & $7 \pm 2$ & $23.0 \pm 0.8$ & $78 \pm 3 *$ & $2 \pm 1$ & $188 \pm 11$ & $88 \pm 7$ & $4: 32 \pm 2: 10$ \\
\hline R8 & $\begin{array}{l}\text { Team } \\
\text { Avg. }\end{array}$ & $7: 20 \pm 2: 55$ & $864 \pm 61$ & $118.8 \pm 61$ & $134 \pm 36$ & $31 \pm 17$ & $5 \pm 2$ & $7 \pm 3$ & $22.9 \pm 0.7$ & $78 \pm 4^{*}$ & $2 \pm 1$ & $188 \pm 7$ & $89 \pm 5$ * & $4: 26 \pm 1: 20$ \\
\hline
\end{tabular}




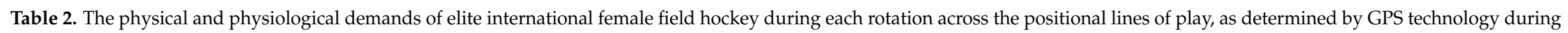
match-play. All data are presented as mean \pm SD.

\begin{tabular}{|c|c|c|c|c|c|c|c|c|c|c|c|c|c|}
\hline & & $\begin{array}{l}\text { Duration } \\
\text { (min:ss) }\end{array}$ & $\begin{array}{c}\text { Total } \\
\text { Distance (m) }\end{array}$ & $\begin{array}{l}\text { High-Speed } \\
\text { Distance }\end{array}$ & $\begin{array}{c}\text { Sprint } \\
\text { Distance }\end{array}$ & $\begin{array}{l}\text { No. of Ac- } \\
\text { celerations }\end{array}$ & $\begin{array}{l}\text { No. of De- } \\
\text { celerations }\end{array}$ & $\begin{array}{l}\text { Peak Speed } \\
\quad(\mathbf{k m} \cdot \mathbf{h})\end{array}$ & $\begin{array}{c}\text { Max Speed } \\
(\%)\end{array}$ & $\begin{array}{c}\text { No. } \\
\text { Sprints } \\
>20 \mathrm{~km} \cdot \mathrm{h}\end{array}$ & $\begin{array}{l}\text { Peak HR } \\
\text { (bpm) }\end{array}$ & $\mathrm{HR}_{\max }(\%)$ & $\begin{array}{c}\text { Time }>80 \% \\
\mathrm{HR}_{\max }(\min : \mathrm{ss})\end{array}$ \\
\hline \multirow{3}{*}{ R1 } & Def & $7: 00 \pm 2: 35$ & $866 \pm 108$ & $118 \pm 44^{\mathrm{b}, \mathrm{c}}$ & $23 \pm 8$ & $5 \pm 1^{b, c}$ & $7 \pm 2^{b, c}$ & $22.5 \pm 0.3^{b, c}$ & $77 \pm 2^{b, c}$ & $2 \pm 1^{b, c}$ & $191 \pm 4^{b, c}$ & $89 \pm 3$ & $4: 20 \pm 1: 26$ \\
\hline & Mid & $7: 00 \pm 2: 30$ & $832 \pm 156$ & $156 \pm 37$ & $33 \pm 8$ & $6 \pm 1$ & $8 \pm 2$ & $23.5 \pm 0.5$ & $79 \pm 2$ & $3 \pm 1$ & $186 \pm 6$ & $89 \pm 6$ & $4: 13 \pm 2: 10$ \\
\hline & Fwd & $7: 00 \pm 2: 30$ & $910 \pm 205$ & $182 \pm 44$ & $44 \pm 15^{b}$ & $7 \pm 2$ & $8 \pm 2$ & $23.5 \pm 0.7$ & $79 \pm 2$ & $3 \pm 1$ & $186 \pm 12$ & $86 \pm 5^{a, b}$ & $4: 26 \pm 2: 25$ \\
\hline \multirow[b]{2}{*}{ R2 } & Def & $7: 15 \pm 2: 10$ & $828 \pm 102$ & $110 \pm 25^{b, c}$ & $23 \pm 8^{b, c}$ & $5 \pm 2^{b, c}$ & $7 \pm 1^{b, c}$ & $22.7 \pm 0.6^{\mathrm{b}, \mathrm{c}}$ & $78 \pm 2$ & $2 \pm 1$ & $192 \pm 5^{\mathrm{b}, \mathrm{c}}$ & $90 \pm 4$ & $4: 33 \pm 1: 20$ \\
\hline & Mid & $7: 15 \pm 2: 10$ & $900 \pm 41^{a, c}$ & $155 \pm 22$ & $32 \pm 67$ & $6 \pm 1$ & $8 \pm 2$ & $23.3 \pm 0.4$ & $79 \pm 3$ & $2 \pm 1$ & $188 \pm 7$ & $90 \pm 6$ & $4: 05 \pm 1: 25$ \\
\hline \multirow{3}{*}{ R3 } & Def & $6: 55 \pm 2: 40$ & $795 \pm 76^{b}$ & $99 \pm 23^{b, c}$ & $21 \pm 7$ & $4 \pm 1^{\mathrm{b}, \mathrm{c}}$ & $7 \pm 2$ & $22.4 \pm 0.6^{b, c}$ & $77 \pm 2$ & $2 \pm 1$ & $192 \pm 5$ & $90 \pm 4$ & $4: 26 \pm 1: 15$ \\
\hline & Mid & $7: 20 \pm 3: 00$ & $868 \pm 68$ & $150 \pm 14$ & $31 \pm 16$ & $6 \pm 2$ & $8 \pm 2^{a, c}$ & $23.0 \pm 1.3$ & $78 \pm 4$ & $2 \pm 1$ & $187 \pm 6$ & $90 \pm 6$ & $4: 22 \pm 1: 30$ \\
\hline & Fwd & $7: 00 \pm 0: 30$ & $858 \pm 195$ & $160 \pm 62$ & $34 \pm 12$ & $5 \pm 2$ & $7 \pm 2$ & $23.3 \pm 0.8$ & $78 \pm 3$ & $2 \pm 1$ & $188 \pm 10$ & $87 \pm 5^{\mathrm{a}, \mathrm{b}}$ & $4: 26 \pm 2: 15$ \\
\hline \multirow{3}{*}{ R4 } & Def & $8: 00 \pm 3: 15$ & $883 \pm 112$ & $105 \pm 24^{b, c}$ & $23 \pm 8^{b, c}$ & $5 \pm 1$ & $7 \pm 2$ & $22.8 \pm 0.7^{\mathrm{b}, \mathrm{c}}$ & $78 \pm 1$ & $2 \pm 1$ & $191 \pm 7^{b, c}$ & $89 \pm 4$ & $4: 39 \pm 1: 30$ \\
\hline & Mid & $7: 10 \pm 2: 50$ & $858 \pm 98$ & $145 \pm 27$ & $34 \pm 13$ & $5 \pm 2$ & $7 \pm 2$ & $23.2 \pm 0.9$ & $78 \pm 5$ & $2 \pm 1$ & $186 \pm 6$ & $89 \pm 6$ & $4: 18 \pm 2: 00$ \\
\hline & Fwd & $7: 10 \pm 3: 00$ & $878 \pm 101$ & $156 \pm 26$ & $31 \pm 11$ & $6 \pm 1 \mathrm{a}, \mathrm{b}$ & $8 \pm 1 \mathrm{a}, \mathrm{b}$ & $23.2 \pm 0.5$ & $78 \pm 3$ & $2 \pm 1$ & $187 \pm 13$ & $86 \pm 6^{a, b}$ & $4: 31 \pm 2: 15$ \\
\hline \multirow{2}{*}{ R5 } & Def & $7: 30 \pm 2: 30$ & $841 \pm 127^{b}$ & $97 \pm 39^{b, c}$ & $23 \pm 15^{b, c}$ & $4 \pm 1^{\mathrm{b}, \mathrm{c}}$ & $6 \pm 3^{b, c}$ & $22.2 \pm 1.1^{b, c}$ & $78 \pm 3$ & $2 \pm 1$ & $189 \pm 6^{\mathrm{b}, \mathrm{c}}$ & $89 \pm 4$ & $4: 25 \pm 1: 20$ \\
\hline & Fwd & $7: 10 \pm 3: 00$ & $864 \pm 143$ & $154 \pm 36$ & $33 \pm 5$ & $5 \pm 1$ & $8 \pm 2$ & $23.0 \pm 0.3$ & $77 \pm 1$ & $3 \pm 1 \mathrm{a}, \mathrm{b}$ & $184 \pm 10$ & $85 \pm 4^{\mathrm{a}, \mathrm{b}}$ & $4: 25 \pm 2: 15$ \\
\hline \multirow{3}{*}{ R6 } & Def & $8: 20 \pm 3: 15$ & $890 \pm 175$ & $113 \pm 46^{\mathrm{b}, \mathrm{c}}$ & $26 \pm 11^{b, c}$ & $5 \pm 1$ & $8 \pm 2$ & $22.9 \pm 1.1$ & $78 \pm 3$ & $2 \pm 1^{b, c}$ & $192 \pm 7^{c}$ & $90 \pm 5$ & $5: 18 \pm 2: 20^{b, c}$ \\
\hline & Mid & $8: 10 \pm 3: 30$ & $887 \pm 88$ & $146 \pm 36$ & $33 \pm 18$ & $5 \pm 1$ & $7 \pm 2^{a, c}$ & $23.4 \pm 1.4$ & $79 \pm 4^{c}$ & $2 \pm 1$ & $186 \pm 6$ & $89 \pm 6$ & $4: 22 \pm 2: 00$ \\
\hline & Fwd & $7: 00 \pm 2: 25$ & $880 \pm 103$ & $142 \pm 35$ & $34 \pm 9$ & $6 \pm 1^{b, c}$ & $8 \pm 1$ & $22.8 \pm 1.2$ & $78 \pm 3$ & $3 \pm 1$ & $186 \pm 10$ & $86 \pm 4^{\mathrm{a}, \mathrm{b}}$ & $4: 28 \pm 2: 15$ \\
\hline \multirow{3}{*}{ R7 } & Def & $8: 25 \pm 4: 00$ & $925 \pm 147 \mathrm{~b}, \mathrm{c}$ & $115 \pm 20^{b, c}$ & $28 \pm 12$ & $5 \pm 2^{b, c}$ & $7 \pm 2$ & $22.5 \pm 1.2^{\mathrm{b}, \mathrm{c}}$ & $79 \pm 3$ & $2 \pm 1$ & $193 \pm 7^{b, c}$ & $90 \pm 5$ & $5: 21 \pm 2: 00^{b, c}$ \\
\hline & Mid & $7: 25 \pm 3: 10$ & $856 \pm 157$ & $151 \pm 37$ & $34 \pm 22$ & $6 \pm 2$ & $7 \pm 3$ & $23.2 \pm 1.1$ & $80 \pm 4^{c}$ & $3 \pm 1$ & $186 \pm 14$ & $89 \pm 9$ & $4: 18 \pm 2: 10$ \\
\hline & Fwd & $6: 25 \pm 3: 15$ & $808 \pm 100$ & $147 \pm 69$ & $35 \pm 24$ & $6 \pm 1$ & $7 \pm 1$ & $23.3 \pm 1.3$ & $76 \pm 4$ & $3 \pm 1$ & $184 \pm 10$ & $85 \pm 5^{\mathrm{a}, \mathrm{b}}$ & $4: 15 \pm 2: 10$ \\
\hline \multirow{3}{*}{ R8 } & Def & $8: 00 \pm 1: 00$ & $870 \pm 218$ & $111 \pm 27^{b, c}$ & $24 \pm 19^{b, c}$ & $5 \pm 2$ & $7 \pm 3^{b, c}$ & $22.9 \pm 0.4$ & $77 \pm 4^{\mathrm{b}, \mathrm{c}}$ & $2 \pm 1$ & $193 \pm 4^{b, c}$ & $91 \pm 3$ & $4: 38 \pm 1: 00$ \\
\hline & Mid & $7: 10 \pm 3: 25$ & $732 \pm 269^{a}$ & $129 \pm 31$ & $32 \pm 13$ & $5 \pm 2$ & $6 \pm 4$ & $22.9 \pm 0.4$ & $80 \pm 4$ & $2 \pm 1$ & $187 \pm 5$ & $90 \pm 6$ & $4: 18 \pm 1: 30$ \\
\hline & Fwd & $7: 30 \pm 3: 10$ & $8698 \pm 105^{b}$ & $160 \pm 19$ & $38 \pm 15$ & $6 \pm 3^{b, c}$ & $8 \pm 2$ & $22.9 \pm 0.4$ & $78 \pm 3$ & $3 \pm 1^{a, b}$ & $184 \pm 11$ & $85 \pm 5^{\mathrm{a}, \mathrm{b}}$ & $4: 29 \pm 2: 15$ \\
\hline
\end{tabular}

a, b, c signifies the positional variation between the defenders (a), midfield (b) and forwards. ${ }^{*}$ represents the significant difference across each rotation when compared to the average rotation output. 


\section{Discussion}

The primary aim of the current observational study was to quantify each rotation running performance and heart rate responses in elite female field hockey players. Secondly, we aimed to quantify the demands of individual rotations across the positional lines of play. Research conducted on elite female field hockey rotational demands is limited [5,11]. Therefore, an examination of the physical and physiological demands during each rotation in elite female field hockey is warranted. The main findings of the current study were that irrespective of position small to large effects were observed for relative total distance during each rotation when compared to average match-play outputs for elite female hockey players, with no significant difference reported across relative high-speed and relative sprint distance. Furthermore, while no difference was reported across the rotations, a significant main effect was seen across the positional lines of play for multiple GPS variables. Finally, it was observed that $\mathrm{HR}_{\max }(\%)$ was significantly different for players across the rotational phases of play.

The data reported within the current study show that regardless of position elite female field hockey players cover a total distance of $868 \pm 132 \mathrm{~m}\left(124.9 \pm 10.7 \mathrm{~m} \cdot \mathrm{min}^{-1}\right)$ with $140 \pm 39 \mathrm{~m}\left(20.1 \pm 4.5 \mathrm{~m} \cdot \mathrm{min}^{-1}\right)$ covered at high-speed across each rotation. When positional lines of play were considered a significant small to moderate difference for relative values of total distance, high-speed distance and sprint distance were observed. Specifically, defenders were reported to have the lowest relative total distance $\left(119.8 \pm 11.0 \mathrm{~m} \cdot \mathrm{min}^{-1}\right)$ when compared to midfield $\left(123.6 \pm 7.6 \mathrm{~m} \cdot \mathrm{min}^{-1}\right)$ and the forwards $\left(131.1 \pm 11 \mathrm{~m} \cdot \mathrm{min}^{-1}\right)$, with these differences small to very large depending on specific positional comparisons (Figure 1). Furthermore, when high-speed running was considered, very large differences were reported across positions with forwards $\left(23.5 \pm 1.2 \mathrm{~m} \cdot \mathrm{min}^{-1}\right)$ shown to cover increased high-speed running when compared to midfielders $\left(21.8 \pm 1.4 \mathrm{~m} \cdot \mathrm{min}^{-1}\right)$ and defenders (14.9 $\pm 1.2 \mathrm{~m} \cdot \mathrm{min}^{-1}$ ), respectively (Figure 2 ). These findings represent a continuation of trends recently reported by McGuinness et al. [3] with respect to quarters of competitive match-play and agree with this literature. It was suggested that during the quarters of competitive match-play elite female field hockey players would cover an average relative distance of $126 \pm 15.3 \mathrm{~m} \cdot \mathrm{min}^{-1}$. Although these results were similar to that recently reported for the average quarter demands, it is important to note that all rotations have been pooled together to give an average rotational demand. Prior to match-play, coaching staff will determine their tactical approach to the opposition. Once this has been agreed, they will often implement specific match strategies such as key playing personnel being on the pitch longer than others. Coaching staff should aim to establish the average rotational demands for each individual during match-play. By knowing these individual averages, practitioners can modify duration and player numbers within small-sided games to best replicate and exceed the positional and individuals match-play demands [16].

Irrespective of positions. elite female field hockey players complete on average eight rotations during competitive match-play. During these individual rotations, players are likely to spend a mean time of $7 \pm 1 \mathrm{~min}$ on their feet. Within these $7 \mathrm{~min}$, players are likely to cover an average relative total distance of $124.9 \pm 10.7 \mathrm{~m} \cdot \mathrm{min}^{-1}$ with a relative high-speed distance of $20.7 \pm 5.8 \mathrm{~m} \cdot \mathrm{min}^{-1}$. When each rotation was considered, a significant main effect was observed for relative total distance $\left(p \leq 0.001 ; \eta^{2}=0.04\right.$; medium), relative high-speed distance $\left(p \leq 0.001 ; \eta^{2}=0.03\right.$; medium), and the number of sprints $\left(p \leq 0.04 ; \eta^{2}=0.01\right.$; small). A significant decrease was reported for relative total distance for R1 and R2 when compared to other rotations completed by players during match-play (Figure 1). Similar to relative total distance, when relative high-speed distance was quantified, a significant decrease was reported when R1 was compared to other rotations during match-play (Figure 2). Our findings are similar to data previously reported in other team sports [17,18]. Previously, Black and colleagues [18] reported that rotation duration influenced the physical activity profile of female Australian football players within the subsequent quarter of play, specifically short (4-6 min) and moderate (7-12 $\mathrm{min}$ ) duration rotations were shown to generate the highest activity profile during 
match-play. During each rotation, forwards maintained the highest relative output when compared to the defenders and midfielders. It is possible that prior knowledge of the team's rotations strategy may be associated with these higher relative outputs. Indeed, forwards typically are required to perform a rotation of 4-9 min, depending on the tactical requirements of the coaching staff, while defenders and midfield players usually complete longer duration rotations (5-11 $\mathrm{min}$ ) during match-play. Schimpchen et al. [19] recently reported that football player's physical output can be more critically impacted by periods of peak high-velocity running. Our results have shown that after R2 no further reduction in locomotor parameters between rotations was reported. It is apparent that after R2 regardless of position, players relative locomotor activity plateaus. Due to their nomadic nature and the speed of the game, it is likely that an element of fatigue or differential positional pacing strategies may be apparent in particular positional lines of play within elite female field hockey $[19,20]$. Given the extent of the reduction in physical performance across the rotations, it is suggested that, during training, coaching staff implement these typical work periods within conditioning drills to replicate and exceed match demands when required.

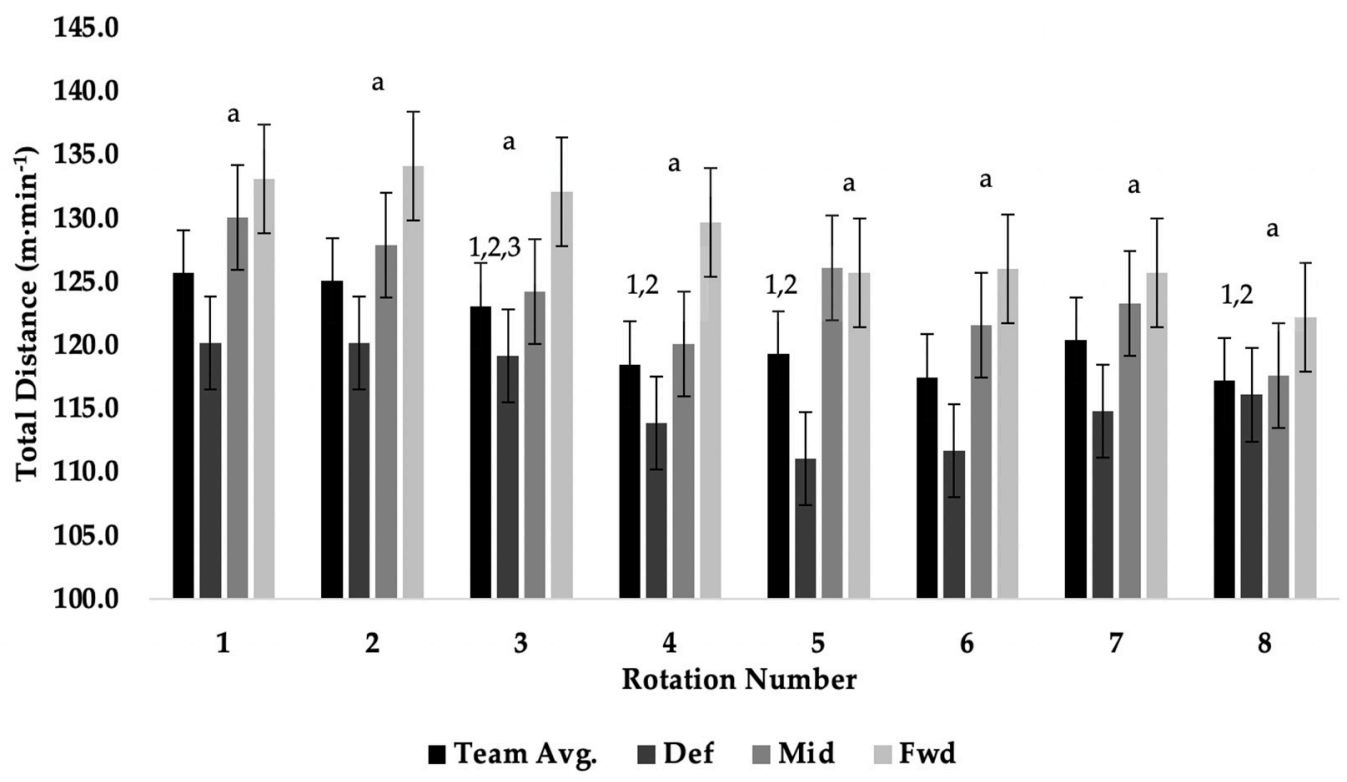

Figure 1. The average relative total distance $\mathrm{m} \cdot \mathrm{min}^{-1}$ of elite female field hockey players across each rotational period during competitive match-play. The letters $a, b, c$ signify the positional variation between the defenders (a), midfield (b) and forwards, while 1, 2, 3, 4, 5, 6, 7, 8 represent the significant difference across each rotation when compared to each other.

Time on feet resulted in players achieving a higher percentage of $\mathrm{HR}_{\max }$ and time above $80 \% \mathrm{HR}_{\max }$ during competitive match-play. Players attained a mean $\mathrm{HR}_{\max }$ of $187 \pm 8 \mathrm{bpm}$ equating to $86 \pm 4 \%$ of $\mathrm{HR}_{\max }$. Regardless of positional group, players were reported to spend $4.29 \pm 1.4 \mathrm{~min}$ of the $7.0 \pm 0.8 \mathrm{~min}$ above $80 \% \mathrm{HR}_{\max }$. Midfielders $\left(90 \pm 6 \%\right.$ ) were reported to reach a higher percentage mean $\mathrm{HR}_{\max }$ during each rotation when compared to the defenders $(90 \pm 6 \%$ ) and forwards ( $86 \pm 4 \%$ ) (Figure 3$)$. Furthermore, our study reported that midfielders spent more time above $80 \% \mathrm{HR}_{\max }(4.39 \pm 1.5 \mathrm{~min})$ when compared to the defenders $(3.54 \pm 1.4 \mathrm{~min})$ and forwards $(4.10 \pm 2.1 \mathrm{~min})$. These results are in line with the findings reported by Harry and Booysen [4], who suggested that players who had a quicker heart rate recovery time had an enhanced ability to cope with the intensity of competitive match-play and could maintain the capacity to run at higher speeds. Forwards were likely to spend the least amount of time on feet during each rotation, had an overall lower mean percentage of $\mathrm{HR}_{\max }$, and could run at a higher intensity. These results suggest that reducing the time midfielders spend in each rotation may, in turn, increase their overall levels of intensity during match-play. Given the above, 
coaches need to develop specific rotational strategies to get the most out of each individual across all the positional lines of play. By implementing such strategies, coaches can aim to reduce the accumulated fatigue that occurs due to the longer duration bouts, and in turn, minimalize the effect on each individual's running performance [21,22].

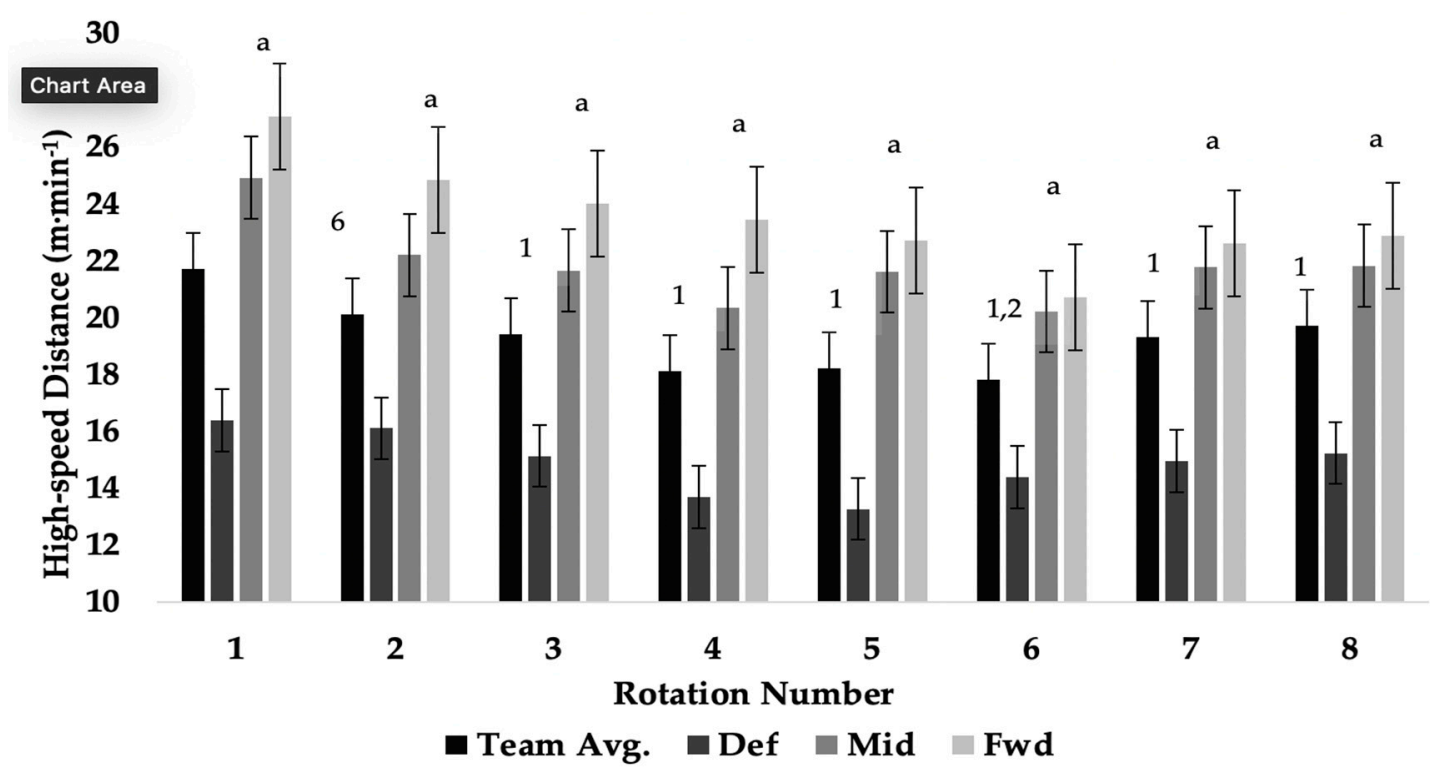

Figure 2. The average relative high-speed distance $\mathrm{m} \cdot \mathrm{min}^{-1}$ of elite female field hockey players across each rotational period during competitive match-play. The letters a, b, c signify the positional variation between the defenders (a), midfield (b) and forwards, while 1, 2, 3, 4, 5, 6, 7, 8 represent the significant difference across each rotation when compared to each other.

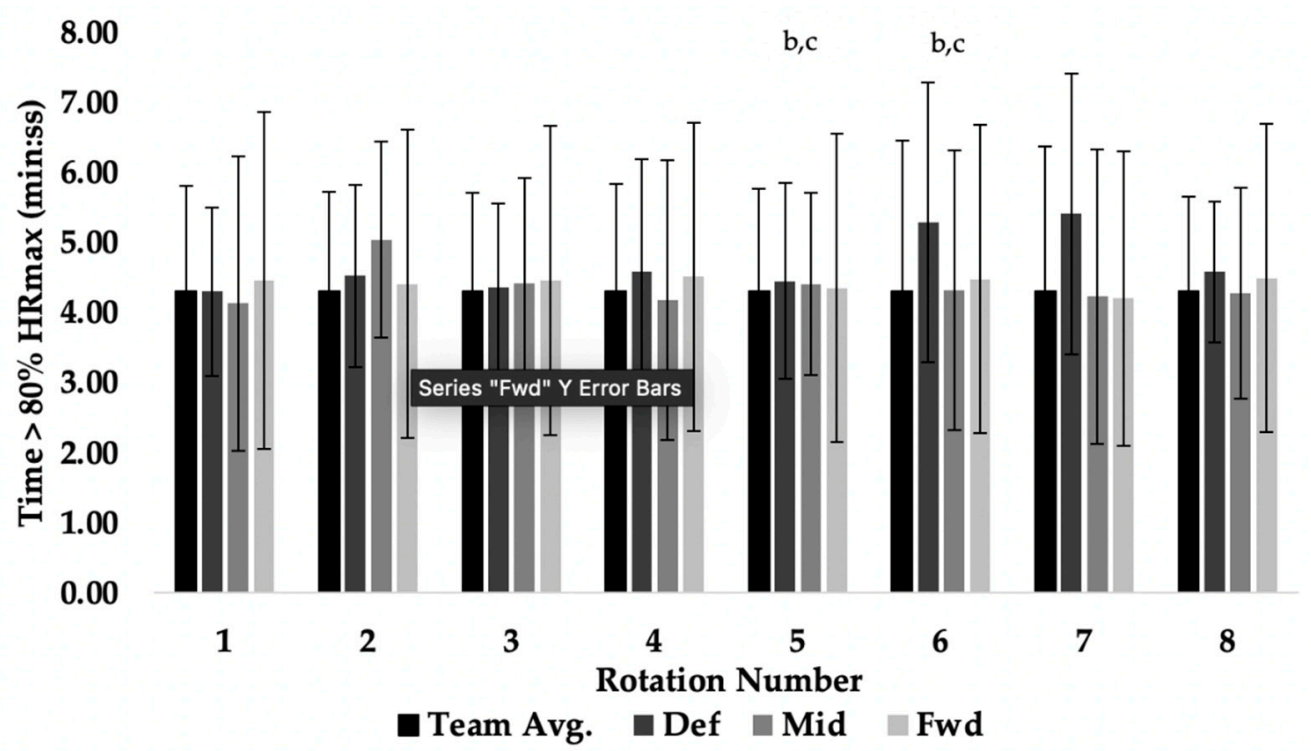

Figure 3. The average time spent $>80 \% \mathrm{HR}_{\max }$ ( $\mathrm{min}$ ) of elite female field hockey players across each rotational period during competitive match-play. The letters a, b, c signify the positional variation between the defenders (a), midfield (b) and forwards.

The differences observed in the physical and physiological performance profiles of elite female hockey players across rotations highlight the potential benefits to coaching staff of knowing these demands of play. Through understanding these rotational demands, 
coaches can better implement rotational and position-specific training drills. Indeed, coaches can manipulate specific drill variables to best target specific physical, technical, tactical, and physiological outcomes that may best replicate competition demand. The results of the investigation need to be interpreted within the context of several limitations. Firstly, although the study included twenty games, the investigation was completed on one elite hockey team, as such, this should be viewed as an applied case study of field hockey rotational demands for the team in question. We, therefore, recommend that practitioners utilise the methodology of the current study to create their team's rotational normative data. Secondly, as we failed to appreciate or understand the analysed team's tactical approach to each game, alongside the standard of opposition faced, these findings could be considered as contextual factors that may impact running performance. We recommend that future research is also focused on understanding how a team's tactical approach and opposition impact the running performance of players during each rotation of play. Finally, given that each players' biological profiles differ in both stature and physical capacity, it is important for authors to acknowledge the application of non-individualised speed thresholds within our analysis. As such, the authors advocate the development of individualised playerspecific running thresholds for elite female hockey [23]. Finally, future research should consider the current advancements in the understanding of team sport activity profiles and the known importance of accelerations and decelerations [24]. Understanding of the acceleration and deceleration demands within elite female hockey is limited, alongside an appreciation of the metabolic power profile of competitive match-play. This added information would aid in determining our comprehension of the energetic cost associated with competitive match-play.

\section{Conclusions}

The current study is the first to report the physical and physiological rotational demands of elite female field hockey players with respect to positional lines of play. The mean rotation duration equated to $7 \pm 0.8 \mathrm{~min}$. During this time, players covered an average distance of $868 \pm 132 \mathrm{~m}$ reflective of $124.9 \pm 10.7 \mathrm{~m} \cdot \mathrm{min}^{-1}$ of which $140 \pm 39 \mathrm{~m}$ or $20.1 \pm 4.5 \mathrm{~m} \cdot \mathrm{min}^{-1}$ was completed at high speed. Relative distance and relative high-speed distance decrease significantly across competitive match-play. Defenders were reported to cover the least amount of relative total $\left(115.9 \mathrm{~m} \cdot \mathrm{min}^{-1}\right)$ and high-speed $\left(14.9 \mathrm{~m} \cdot \mathrm{min}^{-1}\right)$ distance during each rotation. Forwards were reported to cover the most high-speed running $(160 \pm 37 \mathrm{~m})$ when compared to the midfield $(153 \pm 30 \mathrm{~m})$ and defenders $(117 \pm 34 \mathrm{~m})$. Although respective high-speed distances were similar, relative distances were significantly higher. We observed reductions in match-play running across rotations with respect to relative distance, with this being highest between $R 4$ and R5. These rotations were typically observed to occur before and after half time. Practitioners should be aware of this observed drop off in running performance either side of half time and may consider implementing specific performance, nutritional or re-warm up strategies to try and limit the decrease in performance $[25,26]$.

Author Contributions: A.M., D.K. \& K.C. designed the study. Data collection was completed by A.M. and D.K. Data analysis was completed by A.M. Interpretation and manuscript preparation was conducted by A.M., A.G. and K.C. and supported by all authors. All authors have read and agreed to the published version of the manuscript.

Funding: This research received not external funding.

Institutional Review Board Statement: The study was conducted according to the guidelines of the Declaration of Helsinki, and approved by the Institutional Ethics Committee of Technological University Dublin (Approval number; REC-PGR26-201819, Date approved; 25 July 2018).

Informed Consent Statement: Informed consent was obtained for this research.

Conflicts of Interest: The authors declare no conflict of interest regarding this study. 


\section{References}

1. Gabbett, T. GPS Analysis of Elite Women's Field Hockey Training and Competition. J. Strength Cond. Res. 2010, 24, 1321-1324. [CrossRef] [PubMed]

2. Macutkiewicz, D.; Sunderland, C. The Use of GPS to Evaluate Activity Profiles of Elite Women Hockey Players during Match-Play. J. Sports Sci. 2011, 29, 967-973. [CrossRef] [PubMed]

3. McGuinness, A.; Malone, S.; Hughes, B.; Collins, K. The Physical Activity and Physiological Profiles of Elite International Female Field Hockey Players across the Quarters of Competitive Match-Play. J. Strength Cond. Res. Cond. Res. 2018, 1, 1-21. [CrossRef] [PubMed]

4. Harry, K.; Booysen, M. Faster Heart Rate Recovery Correlates With High-Intensity Match Activity in Female Field Hockey Players-Training Implications. J. Strength Cond. Res. 2020, 34, 1150-1157. [CrossRef]

5. Lythe, J.; Kilding, A. The Effect of Substitution Frequency on the Physical and Technical Outputs of Strikers during Field Hockey Match Play. Int. J. Perform. Anal. Sport 2013, 13, 848-859. [CrossRef]

6. McGuinness, A.; Malone, S.; Passmore, D.; Collins, K. Peak Running Intensity of Elite Female Field Hockey Players during Competitive Match Play. J. Strength Cond. Res. 2020. [CrossRef]

7. McMahon, G.; Kennedy, R. Changes in Player Activity Profiles Following the 2015 FIH Rule Changes in Elite Women's Hockey. J. Strength Cond. Res. 2017, 11, 1-24.

8. Vescovi, J.; Klas, A.; Mandic, I. Investigating the Relationships between Load and Recovery in Women's Field Hockey-Female Athletes in Motion (FAiM) Study. Int. J. Perform. Anal. Sport 2019, 19, 672-682. [CrossRef]

9. Bradley, P.; Lago-Peñas, C.; Ezequiel, R. Evaluation of the Match Performances of Substitution Players in Elite Soccer. Int. J. Sports Physiol. Perform. 2014, 9, 415-424. [CrossRef]

10. Delves, R.; Bahnisch, J.; Ball, K.; Duthie, G. Quantifying Mean Peak Running Intensities in Elite Field Hockey. J. Strength Cond. Res. 2019, 1, 1-7. [CrossRef]

11. Linke, D.; Lames, M. Substitutions in Elite Male Field Hockey-A Case Study. Int. J. Perform. Anal. Sport 2017, 16, 924-934. [CrossRef]

12. Murray, A.; Varley, M. Activity Profile of International Rugby Sevens: Effect of Score Line, Opponent, and Substitutes. Int. J. Sports Physiol. Perform. 2015, 10, 791-801. [CrossRef] [PubMed]

13. McGuinness, A.; Malone, S.; Petrakos, G.; Collins, K. The Physical and Physiological Demands of Elite International Female Field Hockey Players during Competitive Match-Play. J. Strength Cond. Res. 2019, 33, 3105-3113. [CrossRef] [PubMed]

14. Kotrlik, J.; Williams, H. The Incorporation of Effect Size in Information Technology, Learning, and Performance Research. Learn. Perform. J. 2003, 21, 1-7.

15. Sawilowsky, S. New Effect Size Rules of Thumb. J. Mod. Appl. Stat. Methods 2009, 8, 597-599. [CrossRef]

16. Riboli, A.; Coratella, G.; Rampichini, S.; Cé, E.; Esposito, F. Area per Player in Small-Sided Games to Replicate the External Load and Estimated Physiological Match Demands in Elite Soccer Players. Hum. Movemant 2020, 21, 100-119. [CrossRef]

17. Hills, S.P.; Radcliffe, J.N.; Barwood, M.J.; Arent, S.M.; Cooke, C.B.; Russell, M. Practitioner Perceptions Regarding the Practices of Soccer Substitutes. PLoS ONE 2020, 15, 1-24. [CrossRef]

18. Black, G.M.; Gabbett, T.J.; Johnston, R.D.; Naughton, G.; Cole, M.H.; Dawson, B. The Influence of Rotations on Match Running Performance in Female Australian Football Midfielders. Int. J. Sport Nutr. Exerc. Metab. 2017, 13, 434-441. [CrossRef]

19. Schimpchen, J.; Gopaladesikan, S.; Meyer, T. The Intermittent Nature of Player Physical Output in Professional Football Matches: An Analysis of Sequences of Peak Intensity and Associated Fatigue Responses. Eur. J. Sport Sci. 2020, 1-21. [CrossRef]

20. Pryor, L.; Johnson, E.; Yoder, H.; Looney, D. Keeping Pace: A Practitioner-Focused Review of Pacing Strategies in Running. Strength Cond. J. 2020, 42, 67-75. [CrossRef]

21. Waldron, M.; Highton, J.; Daniels, M.; Twist, C. Preliminary Evidence of Transient Fatigue and Pacing during Interchanges in Rugby League. Int. J. Sport. Physiol. Perform. 2013, 8, 157-164. [CrossRef] [PubMed]

22. Black, G.; Gabbett, T.; Johnston, R.; Cole, M.; Naughton, G.; Dawson, B. The Influence of Physical Qualities on Activity Profiles of Female Australian Football Match Play. Int. J. Sports Physiol. Perform. 2018, 13, 524-529. [CrossRef] [PubMed]

23. Abt, G.; Lovell, R. The Use of Individualized Speed and Intensity Thresholds for Determining the Distance Run at High-Intensity in Professional Soccer. J. Sports Sci. 2009, 27, 893-898. [CrossRef] [PubMed]

24. Di Prampero, P.; Botter, A.; Osgnach, C. The Energy Cost of Sprint Running and the Role of Metabolic Power in Setting Top Performances. Eur. J. Appl. Physiol. 2015, 115, 451-469. [CrossRef]

25. Fashioni, E.; Langley, B.; Page, R. The Effectiveness of a Practical Half-Time Re-Warm-up Strategy on Performance and the Physical Response to Soccer-Specific Activity. J. Sports Sci. 2019, 38, 140-149. [CrossRef]

26. Russell, M.; Harper, L.; Kilduff, L. Half-Time Strategies to Enhance Second Half Performance in Team Sports Players: A Review and Reccomendations. Sport. Med. 2015, 45, 353-364. [CrossRef] 